\title{
Großzählungsdaten 1991 auf der Basis individueller räumlicher Gebietseinheiten
}

\author{
Erich Wonka \\ Österreichisches Statistisches Zentralamt, Wien
}

\begin{abstract}
Zusammenfassung: Die Großzählungsdaten (Volks-, Häuser- und Wohnungszählung sowie Arbeitsstättenzählung) des Österreichischen Statistischen Zentralamtes (ÖSTAT) werden für administrative Gebietseinheiten (z.B. Gemeinden) und Zählsprengel erstellt. Für viele räumliche Untersuchungen wäre eine Aufbereitung der statistischen Werte nach kleinräumigen Gebietseinheiten (z.B. Gitternetzen, Baublöcken) wünschenswert. Will man für ausgewählte Gebiete statistische Daten der Großzählung unabhängig von den bereits vorgegebenen administrativen Gebietseinheiten in beliebiger Form regional zusammenfassen, ist dies nur über das Gebäuderegister des ÖSTAT möglich. Voraussetzung dabei ist, daß jedem adressierten Gebäude im Gebäuderegister eine Koordinate zugeordnet wird. Im ÖSTAT ist mit Unterstützung des Bundesamtes für Eich- und Vermessungswesen (BEV) geplant, die Gebäudekoordinaten der digitalen Katastralmappe in das Gebäuderegister zu übernehmen.
\end{abstract}

\begin{abstract}
Data of the Combined Census (Population Census, Housing Census and Census of Non-Agricultural Local Units of Employment) of the Austrian Central Statistical Office (ÖSTAT) are available for administrative units (for instanc Commune) and enumeration districts. For many analyses, it can be desirable to process such data according to smaller units, for example housing blocks or grid-maps. If one wishes to assemble data on a regional basis different from that pro-selected, it is necessary to make use of the building register of the ÖSTAT. The pre-requisite is that every building in the building register is to be assigned a coordinate. The ÖSTAT, with the support of the Federal Office of Metrology and Surveying (BEV), is planning to incorporate the building coordinates of the digital land-registry map into the building register.
\end{abstract}

\section{Zur Problematik des räumlichen Vergleichs bei Ge- bietseinheiten}

Sollten in der Statistik Massenerscheinungen verglichen werden, so wird an sie die Forderung gestellt, daß sie vergleichbar sind; insbesondere müssen sie dem Gesetz der großen Zahl genügen, damit Zufallsergebnisse ausgeschaltet werden können; sonst ist der Vergleich bedeutungslos. Beim Vergleich statistischer Massen sollen auch die Be- 
obachtungsmassen wenigstens annähernd gleich groß sein. Im Gegensatz zur Statistik besteht das Wesen von räumlichen Untersuchungen in der Geographie und thematischen Kartographie gerade darin, die statistischen Angaben zu vereinzeln (vgl. dazu WITT, 1972). In der Statistik stehen sachliche und zeitliche Gruppierungen im Vordergrund, bei der Geographie dagegen die regionalen. Die Art der regionalen Gliederung in Gebietseinheiten ist somit von großer Bedeutung. Unter Gebietseinheiten werden hier solche Einheiten verstanden, auf die quantitative Angaben, in erster Linie statistische Daten, ohne genauere Lokalisierbarkeit bezogen sind. Welche Gebietsgliederung dann z.B. bei der kartographischen Darstellung statistischer Daten eine optimale Aussage gewährleisten, wird vom Ziel der Untersuchung abhängig sein. In vielen Fällen greift man nur deshalb auf administrative Gebietsgliederungen zurück, weil die statistischen Daten nur nach diesen räumlichen Einheiten vorhanden sind.

Für viele räumliche Untersuchungen sind administrative Gebietseinheiten ungenügend. Man übersieht dabei leider in den meisten Fällen die aus der Ungleichheit dieser Einheiten sich ergebenden Folgen, insbesondere im Hinblick auf die räumliche Vergleichbarkeit. Ein Problem ist, daß die Flächengrößenunterschiede derartiger Gebietseinheiten sehr groß sind. So ist der Variationskoeffizient, der ausdrückt, um wieviel die Gebietseinheiten von der durchschnittlichen Gebietseinheit abweichen, bei den Gemeinden $105 \%$ und bei den statistischen Zählsprengel sogar 205\%. Eine weitere Ursache liegt darin, daß keine individuellen Homogenitätswünsche berücksichtigt werden (die Homogenitätswünsche sind vom Untersuchungszweck abhängig) und auch der Grenzverlauf administrativer Gebietseinheiten sehr unregelmäßig ist. Was die Forderung der Homogenität angeht, so sollen homogene Gebiete in vielen Fällen erst durch die Untersuchung überhaupt erst ermittelt werden. Die ungleiche Form und Größe führen bei räumlichen Untersuchungen von statistischen Daten zu Vergleichsstörungen bei der Interpretation. Bereits WiTT (1972) wies darauf hin, daß bei dem räumlichen Vergleich und allen damit verbundenen kartographischen Maßnahmen vor allem die Flächengrößenunterschiede mit berücksichtigt werden müssen. Vernachlässigt man diesen Gesichtspunkt, so sind die statistischen Berechnungen allein ziemlich wertlos und die räumlichen Ergebnisse äußerst dubios.

Diese kurz angerissene Problematik der Vergleichbarkeit bei räumlichen Untersuchungen zeigt, wie wichtig es ist, statistische Daten auch nach kleinräumigen Gebietseinheiten (z.B. Gitternetze, Baublöcke) zu bekommen.

\section{Das Gebäuderegister im ÖSTAT}

Im ÖSTAT ist geplant, für die Großzählungsdaten eine allgemeine Lösung zur Bildung flexibler und damit auch kleinräumiger statistischer Gebietseinheiten zu finden. Die Grundlage dazu ist das Gebäuderegister des ÖSTAT und die Grundstücksdatenbank und die digitale Katastralmappe (DKM) des BEV.

Das Gebäuderegister (siehe Abbildung 1) enthält die Anschrift sämtlicher dem ÖSTAT bekannten Gebäudeobjekte. Gebäude sind freistehende oder - bei zusammenhängender 
Bauweise - klar gegeneinander abgegrenzte Baulichkeiten, deren verbaute Fläche mindestens 20 Quadratmeter beträgt.

Auszug aus dem Gebäuderegister des ÖSTAT:

\begin{tabular}{|llllllllll|}
\hline Geb & Wo & EW & Arb & PLZ & STRNAME & HN & GEM & ZSP & OBJNR \\
\hline 1 & 1 & 0 & 0 & 3400 & Burgstraße & 19 & 32408 & 000 & 0645021 \\
1 & 2 & 3 & 2 & 3400 & Schießstattg. & 14 & 32408 & 000 & 0645173 \\
\hline
\end{tabular}

Abbildung 1: Die Gebäudeadressen sind alphabetisch geordnet. Jedes Gebäude hat eine Objektnummer (OBJNR), welche innerhalb Österreichs von 1 bis n reicht. Diese Objektnummer stellt die Verbindung mit den Großzählungsdaten her. Die kleinste statistische Erhebungseinheit, die man mit den Großzählungsdaten herstellen kann, ist somit die Gebäudeadresse. Weiters enthalten die Adressen die Gebäude-, Wohnungs, Einwohner- und Arbeitsstättenzahl, Gemeindenummer Zählsprengelkennziffer usw.

\subsection{Gebäuderegister erweitert um die Hausnummernkoordinaten}

Ordnet man nun jedem adressierten Gebäude in dem Gebäuderegister die entsprechenden Koordinaten zu, erhält die Statistik jene Flexibilität für die räumliche Aufbereitung, die es ihr ermöglicht, allen an sie gestellten Anforderungen gerecht zu werden. Ein Lösungsansatz, wie dieser Weg beschritten werden kann, wird an Hand eines kleineren Probegebietes gezeigt. Nur über das Gebäuderegister, der Grundstücksdatenbank und unter Zuhilfenahme der digitalen Katastralmappe (DKM) ist eine gebäudeweise Verortung der Großzählungsdaten möglich.

Der digitale Katasterplan enthält zwar nicht die Gebäudeadressen, dafür aber die Grundstücksnummern der Gebäude oder - wenn diese nicht vorhanden sind - die Nummern der Grundstücke, auf dem die Gebäude stehen. Da das Grundstücksverzeichnis sowohl die Adressen als auch die dazugehörigen Grundstücksnummern enthält, kann die entsprechende Hausnummer im digitalen Katasterplan ausgewiesen werden. (siehe Abbildung 2). Sind dann die Hausnummern (ON) im Katasterplan digital erfaßt, kann man die Hausnummernkoordinaten in das Gebäuderegister des ÖSTAT übertragen (siehe Abbildung 3). Welchen Koordinatenwert man zur Lagebezeichnung des Gebäudes nimmt, ist kaum von Bedeutung. Dabei muß sich der Koordinatenwert nicht am Gebäudemittelpunkt orientieren. Für statistische Zwecke reicht es auch aus, wenn irgend ein Eckpunkt des Gebäudes oder wie in diesem Fall die Position der Hausnummern im Katasterplan als Koordinatenwert herangezogen wird.

Der größte Teil der Adressen des Gebäuderegisters und des Grundstücksverzeichnisses stimmen überein. Ist dies nicht der Fall, müssen die Adressen aufeinander abgestimmt werden. 
Digitaler Katasterplan erweitert um Hausnummern des Grundstückverzeichnis:

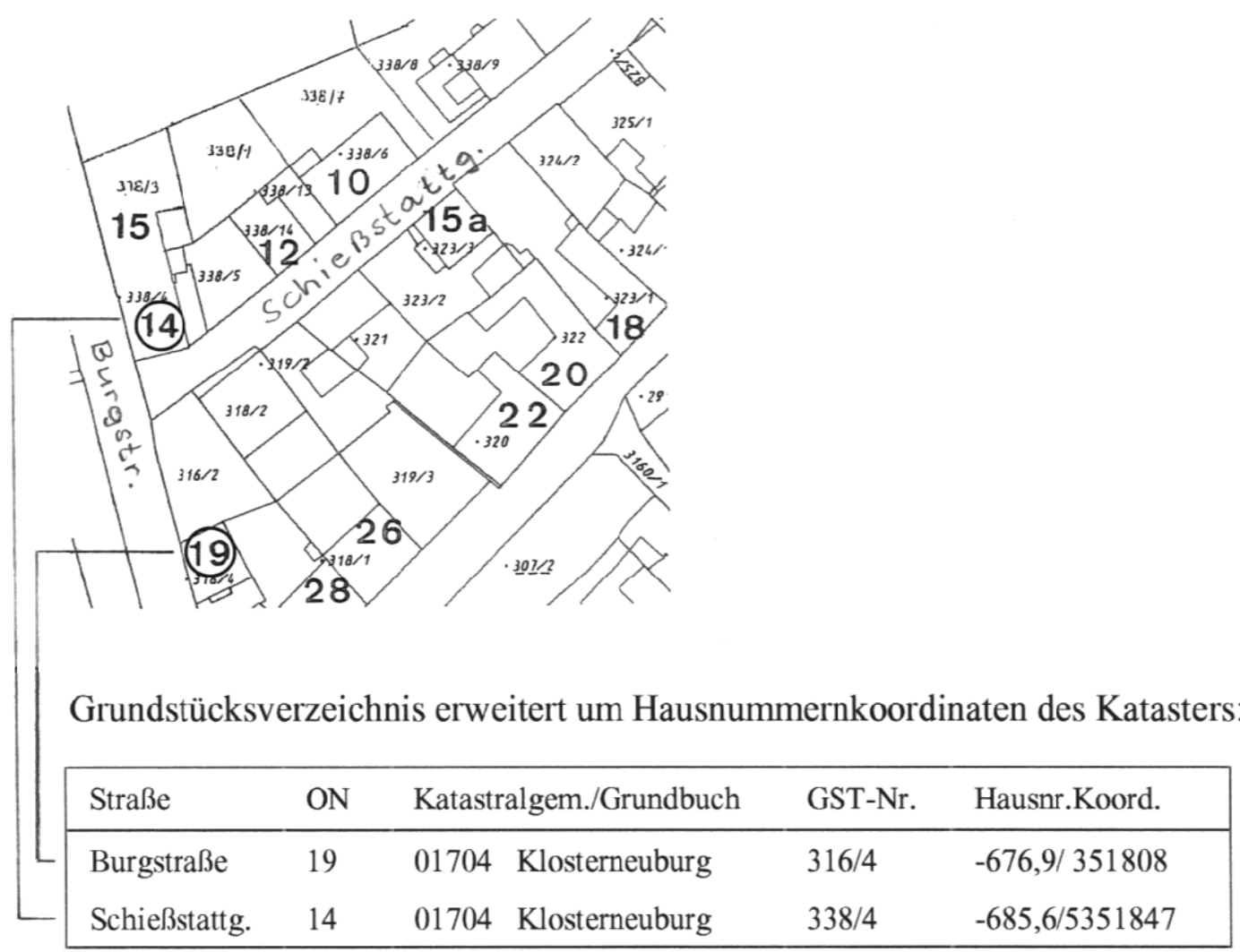

Abbildung 2: Der digitale Katasterplan (siehe oben) wurde um die Hausnummern (hier Orientierungsnummern oder ON) und das Grundstücksverzeichnis (siehe unten) um die Gauß-Krüger Koordinaten der Hausnummern (=Gebäudekoordinaten) erweitert.

Gebäuderegister erweitert um die Hausnummernkoordinaten, KG-Nr. u. GST-Nr.:

\begin{tabular}{|lllllllll|}
\hline Proj & STRNAME & HN & GEM & ZSP & OBJNR & KG & Gst-Nr & Hausnr.Koord. \\
\hline$\ldots . .$. & Burgstraße & 19 & 32408 & 000 & 0645021 & $\mathbf{1 7 0 4}$ & $\mathbf{3 1 6 / 4}$ & $\mathbf{- 6 7 6 , 9 / 3 5 1 8 0 8}$ \\
$\ldots . .$. & Schießstattg. & 14 & 32408 & 000 & 0645173 & $\mathbf{1 7 0 4}$ & $\mathbf{3 3 9 / 4}$ & $\mathbf{- 6 8 5 , 6 / 3 5 1 8 4 7}$ \\
\hline
\end{tabular}

Abbildung 3: Der Adreßteil des Gebäuderegisters beginnt mit einem freien Feld, in dem später die Eingabe des Projektgebietcodes erfolgt (siehe Abbildung 5 und 6). Die folgenden Spalten enthalten aus Platzgründen nur einige Merkmale wie Straßennname, Hausnummer, Gemeinde- und Zählsprengelkennziffer, Objektnummer. Der Adreßteil des Gebäuderegisters wurde dann um die Hausnummernkoordinaten des digitalen Katasterplanes erweitert (letzte Spalte). Zum Zwecke der Lagekontrolle empfiehlt es sich, aus dem Grundstücksverzeichnis auch die Katastralgemeindenummer (KG-Nr.) und die Grundstücksnummer (GST-Nr) des Gebäudes in das Gebäuderegister zu übernehmen. 


\subsection{Bildung von individuellen Gebietseinheiten (Projektgebieten)}

Sind nun die Gebäudekoordinaten im Gebäuderegister des ÖSTAT zu den Gebäudeadressen eingespeichert, ist ihre geographische Position eindeutig festgelegt. Damit kann das statistische Datenmaterial auf jede gewünschte Bezugseinheit aggregiert werden. Welche Arbeitsschritte dazu notwendig sind, wird im folgenden beispielhaft gezeigt.

Um die Großzählungsdaten mit Hilfe des Programms PROJEKTGEBIETE aggregieren zu können, müssen die in das jeweilige Projektgebiet fallenden Gebäude zusammengefaßt werden. Egal wie die Gebietsbildung später erfolgt (z.B. als Gitternetz oder Baublock), müssen vorerst die Hausnummernkoordinaten mit den entsprechenden Hausnummern digital als 'Hausnummernkarte' ausgedruckt werden (siehe Abbildung 4a). Wählt man als Projektgebiet Gitternetze, so bietet sich das Gauß-Krüger-Netz an. Die Gitternetze werden mit einem Code versehen (siehe Abbildung 4b). Dieser darf maximal 5-stellig sein. Nach der Gitternetzeinteilung werden alle innerhalb einer Gitternetzmasche liegenden Gebäudekoordinaten dieser zugeordnet (siehe Abbildung 4c).

(a)

Attributfile (hier
Hausnr.Koord. von der
Punktcoverage

$+\quad$ Attributfile (hier Netzma-

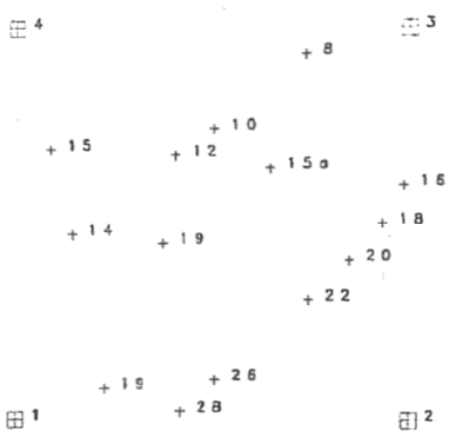
schennr.) von der Polygoncoverage

(b)

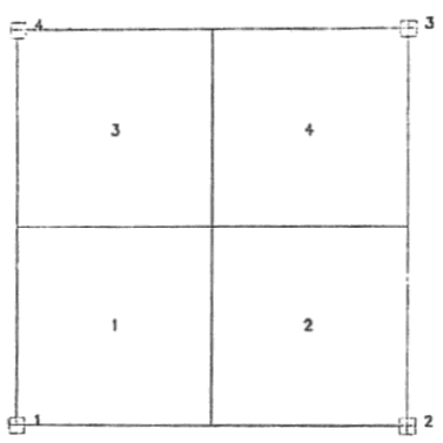

(c)
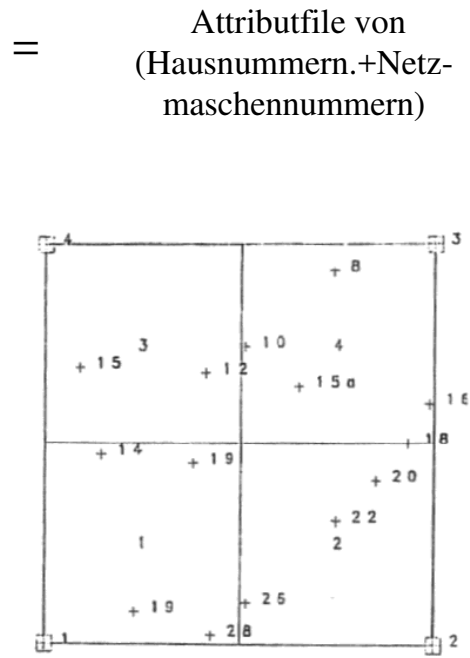

Abbildung 4: Die Abgrenzung von Gitternetzen als Gebietseinheit ist mit Hilfe von GIS-Systemen (z.B. ARC-INFO) leicht zu realisieren. Die Kombination des Punktcoverage der Hausnummernkoordinaten (a) mit dem Polygoncoverage der Gitternetzmaschen (b) geschieht im Arc-Info mit dem Befehl: INTERSECT COVPKT COVPOLY COVNEU POINT. In dem neuen Coverage (c) sind dann alle Attribute der Punkt- und Polygoncoverage und somit auch der Haus- und der Netzmaschennummern enthalten.

Die im Gebäuderegister bereits vorhandenen Merkmale wie Zahl der Gebäude, Wohnungen, Einwohner und Arbeitsstätten (siehe Abbildung 1) können dann sofort auf die Quadrate aufsummiert und kartographisch dargestellt werden. Bei diesen beeits im Ge- 
bäuderegister enthaltenen statistischen Daten erspart man sich somit die Bildung von Projektgebieten. Alle übrigen Großzählungsdaten, die nicht im Gebäuderegister enthalten sind, können nur über den Umweg der Objektnummer auf die gewünschten Gebietseinheiten aggregiert werden. Weitere Bearbeitungsschritte sind notwendig. Dazu muß zunächst die für jedes Gebäude ermittelte Gitternetzmaschennummer (=Projektgebietsnummer) in das Gebäuderegister eingetragen werden (siehe Abbildung $5)$.

Digitaler Katasterplan mit dem Gauß-Krüger-Netz (50m x 50m)

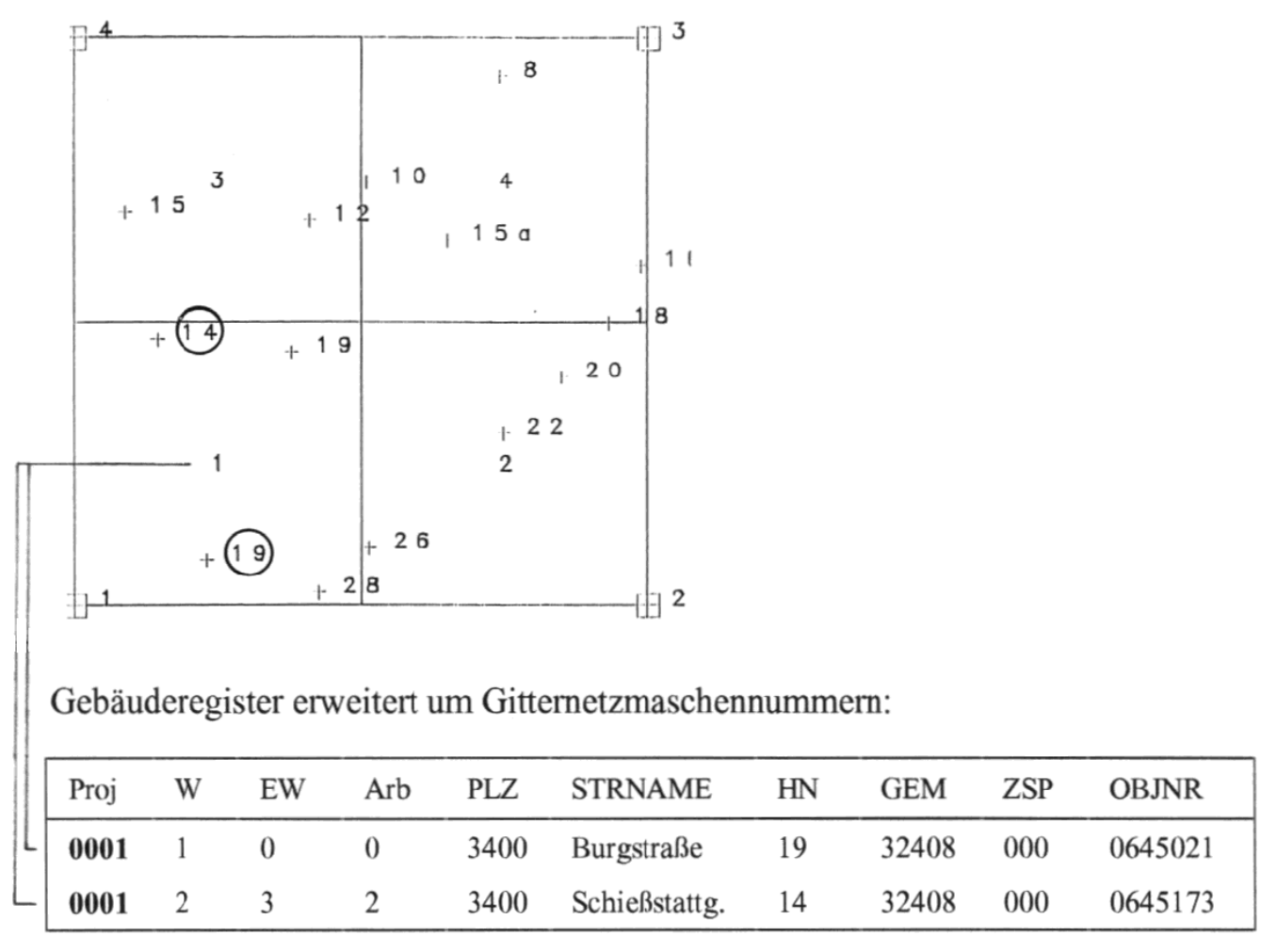

Abbildung 5: Das vom ÖSTAT gelieferte Gebäuderegister beginnt mit einem freien Feld, in dem vom externen Benutzer jetzt die Eingabe des Projektgebietcodes (hier Gitternetzmaschennummer) erfolgt (vgl. auch Abbildung 3).

Ähnlich wie bei der Bildung von Gitternetzmaschen geht man bei der Bildung von unregelmäßigen Gebietsabgrenzungen, wie z.B. bei Baublöcken, vor. Anstelle der Gitternetzmaschennummer tritt hier die Baublocknummer. Alle in einem Baublock liegenden Gebäude werden diesem Baublock zugeordnet, indem sie dieselben Baublocknummern erhalten. Diese werden dann im Gebäuderegister zu den entsprechenden Gebäudeadressen eingetragen (siehe Abbildung 6). 
Digitaler Katasterplan ergänzt um Baublöcke:

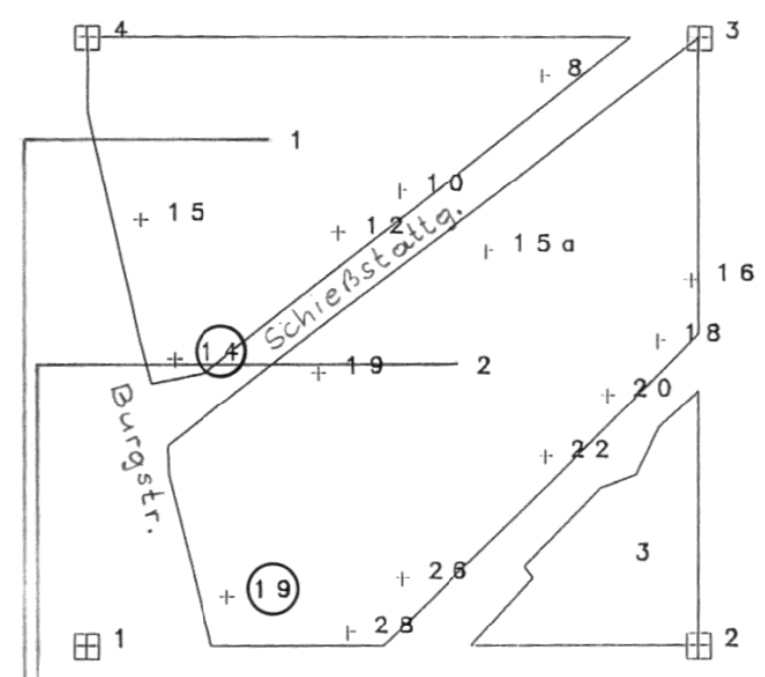

Gebäuderegister erweitert um Baublocknummern:

\begin{tabular}{|llllllllll|}
\hline Proj & Wo & EW & Arb & PLZ & STRNAME & HN & GEM & ZSP & OBJNR \\
\hline $\mathbf{0 0 0 2}$ & 1 & 0 & 0 & 3400 & Burgstraße & 19 & 32408 & 000 & 0645021 \\
\hline $\mathbf{0 0 0 1}$ & 2 & 3 & 2 & 3400 & Schießstattg. & 14 & 32408 & 000 & 0645173 \\
\hline
\end{tabular}

Abbildung 6: In das vom ÖSTAT gelieferte Gebäuderegister wird vom externen Benutzer der Code für die Projektgebiete (hier Baublocknummern) in die 1. Spalte eingetragen.

$\mathrm{Zu}$ beachten ist, daß aufgrund der Geheimhaltungsbestimmung für die Großzählungsdaten eine Mindestaggregation erforderlich ist. Die individuellen Bezugseinheiten müssen vom externen Benutzer so gebildet werden, daß sie mindestens 4 Gebäude umfassen, wenn Daten aus der HWZ abgefragt werden. Zusätzlich zu dieser Anforderung muß eine Bezugseinheit mindestens 30 Einwohner beinhalten, wenn Daten aus der VZ benötigt werden. Für viele räumliche Untersuchungen (z.B. bei Dorfuntersuchungen) ist aber eine Mindestaggregation von 30 Einwohnern viel zu hoch (vgl. dazu AUFHAUSER, 1996). Die Mindestaggregation sollte höchstens 20 Einwohner betragen um damit auch in ländlichen Gebieten einigermaßen sinnvoll arbeiten zu können.

Der erste Bearbeitungsschritt im ÖSTAT besteht in einer Prüfung der von ihnen übermittelten Diskette bezüglich einiger Anfoderungen wie z.B.:

- Projektgebiete mit weniger als 4 Objekten

- Projektgebiete mit weniger als 30 Einwohnern wenn VZ-Daten benötigt werden

- Übereinstimmung der Objektnummern auf der Diskette mit dem Gebäuderegister

- Keine Mehrfachzuordnung von Objekten zu Projektgebieten. 


\section{Abfrage der Großzählungsdaten für individuelle Gebietseinheiten}

Nachdem die Gebietsbildung abgeschlossen ist (d.h. die Objektnummern wurden für die entsprechenden Gebiete zusammengefaßt), erfolgt die eigentliche Abfrage der Merkmale aus den folgenden Basisbeständen der Großzählung.

112 und 113 Basisbestände mit den Daten der HWZ

1143 Basisbestand mit den Daten der VZ

1120, 1121, 1170 - 1173 Basisbestände mit den Daten der AZ

Die Abfrage von Daten aus diesen Basisbeständen wird mit den Automationsprogrammen TABGEN oder TACO durchgeführt. Während beim TABGEN Programm nur Merkmalskombinationen innerhalb eines Basisbandes abgefragt werden können, sind beim TACO Programm keine Einschränkungen vorhanden.

Der externe Benutzer wählt aus diesen Basisbeständen die gewünschten Daten für die von ihm definierten Projektgebiete aus und gibt diese dem ÖSTAT in der von ihm gewünschten Merkmalanordnung weiter (siehe Abbildung 7). Als Ergebnis erhält dann der externe Benützer die Merkmale auf Band oder auf Papier (siehe Abbildung 8).

\begin{tabular}{|lccc|}
\hline Projketgebiete & $\begin{array}{c}\text { Zahl der Woh- } \\
\text { nungen mit } \\
\text { Hauptwohnsitz }\end{array}$ & $\begin{array}{c}\text { Zahl der Woh- } \\
\text { nungen mit } \\
\text { Zweitwohnsitz }\end{array}$ & $\begin{array}{c}\text { Zahl der Woh- } \\
\text { nungen insge- } \\
\text { samt }\end{array}$ \\
\hline 1.0001 & & & \\
2.0002 & & & \\
$3 \ldots \ldots .$. & & & \\
\hline
\end{tabular}

Abbildung 7: Bekanntgabe der Großzählungsmerkmale für die Projektgebiete vom externen Benutzer.

„Tabelle 1: Zahl der Wohnungen mit Haupt- und Zweitwohnsitz“;;;

„Projektgebiete“;Wohnungen mit HWS“;Wohnungen mit ZWS“;Wohnungen insgesamt“;

„Proj 00001“; $11 ; 3 ; 14$

„Proj 00002“; $37 ; 11 ; 48$

„Proj 00003“; $55 ; 14 ; 69$

Abbildung 8: Die Großzählungsdaten werden vom ÖSTAT für die Projektgebiete auf Diskette im „ASCII Delimited“-Format geliefert.

Das ÖSTAT bewahrt die entsprechenden EDV-Bestände 3 Monate ab dem Lieferdatum noch auf. Auch die Zuordnung der Objekte zu den vom Benützer definierten Projektge- 
bieten wird im ÖSTAT nach 3 Monaten gelöscht. Sollte der Benützer Folgeaufträge beabsichtigen, muß sich dieser ein Duplikat der Diskette mit dem Auszug aus dem Gebäuderegister mit den entsprechenden Projekt-Codes aufbewahren.

\section{Statistische Auswertungen auf der Basis von Pro- jektgebieten}

Für statistische Auswertungen der Großzählungsdaten bei einer Stadtgliederung kommen Statistische Zählsprengel, Stadtteile, Baublöcke und Gitternetze in Frage. Sieht man von den Statistischen Zählsprengeln ab, können all diese räumlichen Bezugseinheiten nur mit Hilfe von Projektgebieten gebildet werden. Vor allem die Gitternetze gestatten sowohl einen Flächenvergleich als auch eine Zusammenfassung zu größeren Gebietseinheiten. In den vorliegenden zwei Beispielen wurden deshalb die Großzählungsdaten auf der Basis von 250m großen Gitternetzen untersucht.

Die Repräsentanz der einzelnen Gruppen in einem Gebiet wurde im ersten Beispiel über den Standortquotienten bestimmt (siehe Abbildung 9). Dieser Standortquotient (oder Lokalisationsindex) dient zur Messung von über- oder unterdurchschnittlichen Anteilen von Bevölkerungsteilgruppen in einer Bezugseinheit im Vergleich zum entsprechenden Durchschnitt des Gesamtgebietes. Übersteigt der Wert des Indexes die Zahl 1, liegt ein überdurchschnittliches Vorhandensein einer Sozialgruppe vor. Bei einem Wert unter 1 ist die Sozialgruppe eines Teilraumes im Vergleich zum Gesamtraum unterrepräsentiert.

Das Ausmaß der ungleichen Verteilung von Bevölkerungsteilgruppen über ein Gebiet kann man auch durch den Segregationsindex (IS) ausdrücken. Die Spannweite reicht von 0 bis 100. D.h. bei 0 ist keine räumliche Segregation und bei 100 ist eine vollständige Segregation gegeben. Da dieses statistische Verfahren die Daten in ihrem räumlichen Zusammenhang untersucht, können auch Rückschlüsse auf das gewählte Bezugssystem gezogen werden. Man erkennt, daß es zu unterschiedlichen Rechenergebnissen kommt, je nachdem ob ein Gebiet in Quadrate oder in die Statistische Zählsprengel untergliedert. Aus der Abbildung 10 geht hervor, daß die Selbständigen und Arbeiter in stärkerem Ausmaß segregiert wohnen, während im Vergleich dazu die Angestellten gleichmäßiger über das Stadtgebiet verteilt sind. Die im Vergleich zu den Zählsprengel größere Homogenität der Planquadrate wird durch den höheren Segregationsindex ersichtlich. 


\section{Klosterneuburg}
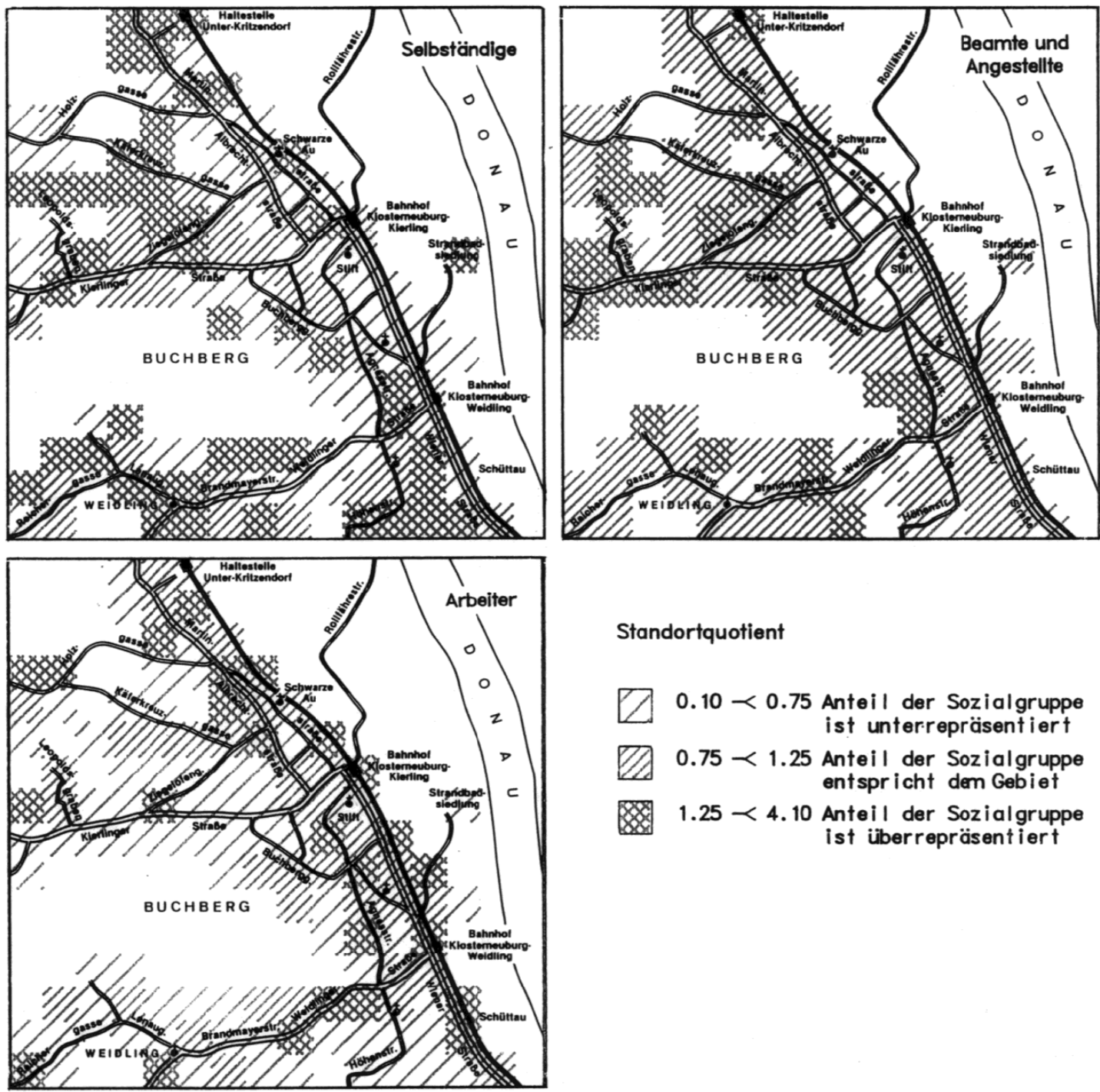

Standortquotient

$0.10 \prec 0.75$ Anteil der Sozialgruppe ist unter-repräsentiert

$0.75 \prec 1.25$ Anteil der Sozialgruppe entspricht dem Gebiet

$1.25 \prec 4.10$ Anteil der Sozialgruppe ist überrepräsentiert

Abbildung 9: Standortquotient auf der Basis von Projektgebieten (hier 250 x 250m große Gitternetzmaschen). Der Standortquotient mißt, inwieweit die Sozialgruppe im Vergleich zum Gesamtgebiet in einem Quadrat über- oder unterrepräsentiert ist. Ist der Standortquotient 1 so entspricht die Sozialgruppe im Quadrat der Repräsentanz im Gesamtraum. 


\begin{tabular}{|lcc|}
\hline Stellung im Beruf & nach Quadrate & nach Zählsprengel \\
\hline Arbeiter & 28,8 & 14,9 \\
Selbständige & 26,3 & 12,4 \\
Angestellte & 23,1 & 11,6 \\
\hline
\end{tabular}

Abbildung 10: Segregationsindex differenziert nach der Stellung im Beruf. Klosterneuburg wurde einmal in $250 \times 250 \mathrm{~m}$ große Quadrate und ein zweites Mal in Statistische Zählsprengel untergliedert.

\section{Literatur}

AUfHAUSER, S. (1996). ÖSTAT-Daten in der örtlichen Raumplanung. Abschlußprojekt für den 2. Hochschullehrgang für Geoinformationswesen an der TU-Wien.

ÖSTERREICHISCHES STATISTISCHES ZENTRALAMT (1991). Benützerhandbuch zur Häuserund Wohnungszählung, Volkszählung (2 Bände) und Arbeitsstättenzählung.

ÖSTERREICHISCHES STATISTISCHES ZENTRALAMT (1994). Projektgebiete.

WITT, W. (1972). Ungelöste Probleme in der thematischen Kartographie. In: Internationales Jahrbuch für Kartographie. S 11-27.

WonkA, E. (1989). Das Gebäuderegister als Grundlage für die Aufbereitung statistischer Daten auf der Basis von kleinräumigen territorialen Einheiten. In: Berichte und Informationen, Nr. 15, Hrsg. Österreichische Akademie der Wissenschaften, Institut für Kartographie, Wien. 19 Seiten.

WonkA, E. (1990). Planquadrate als Bausteine einer sozialräumlichen Stadtgliederung. In: Österreichische Zeitschrift für Statistik und Informatik. 20. Jg., Heft 1-2. Seite 91-109.

WonKA, E. (1993). Die Stellung des Gebäuderegisters innerhalb eines Geographischen Informationssystems (GIS). In: Österreichische Zeitschrift für Vermessungswesen und Photogrammetrie. Wien, Heft 2. 10 Seiten.

Adresse des Autors:

Mag. Dr. Erich Wonka

Österreichisches Statistisches Zentralamt

Technisch-Methodische Abteilung

Hintere Zollamtsstraße 2b

A-1033 Wien 